\section{Addressing a dual public health emergency: supporting physicians to prescribe opioid medications}

Tyndall's article provides timely insight regarding the overdose epidemic. ${ }^{1}$ The coronavirus disease 2019 (COVID-19) pandemic poses a particular unprecedented risk to Canadians with opioid use disorder, who - already vulnerable and marginalized - are heavily dependent on face-to-face health care delivery.

With respect to COVID-19 prevention, the Canadian government defines a category of clinically vulnerable people in which those with "mild to moderate respiratory disease" and those with a "weakened immune system as the result of certain conditions or medicines they are taking" are advised to take extra care. ${ }^{2}$ Accordingly, patients with chronic opioid dependence should be included in the clinically vulnerable high-risk group. This means that sensible health care policies are needed to support people who are dependent on opioids. Because of the COVID-19 pandemic, they are facing a severe shortage of clean opioids. Rapid and coordinated action on the part of the health care community is needed to mitigate the risks of disrupted care for patients with chronic opioid dependence, including the supply of clean opioids.

The pandemic has reversed systemlevel gains in expanding access to medication for opioid use disorder and halted critical opioid research to advance prevention and care. Opioid overdoserelated problems are on the rise for many reasons. ${ }^{1}$ The situation is compounded by the unavailability of bystanders, in some contexts, to administer naloxone, given the need for social distancing and fear of exposure to severe acute respiratory syndrome coronavirus 2 .

Tyndall makes the important point that physicians in British Columbia have been cautious in prescribing opioid medications despite clinical guidance being provided during the COVID-19 pandemic. $^{1}$ The cautious approach to prescribing opioid medications may be the result of messages physicians have become accustomed to, ${ }^{3}$ specifically messages that stigmatize opioids and instill fear in prescribing physicians that their patients may become addicted and ultimately succumb to their addiction. ${ }^{3}$ It is important to address the discomforts and challenges that physicians have in regard to prescribing opioids and encourage physicians to consider additional factors such as socioeconomic status, mental health and substance use problems when having conversations about opioids with patients. ${ }^{4}$

We suggest that further resources be created to support physicians in becoming more comfortable prescribing opioid medications, given that most physicians lack proper training to optimally prescribe opioids. ${ }^{5}$

Recent guidance from the British Columbia Centre on Substance Use, which addresses both the overdose and COVID-19 crises, provides clinical support for prescribing health care professionals, including valuable case studies and microinduction protocols. ${ }^{6}$ However, additional resources to support health care professionals in reflecting on their positionality in regard to prescribing opioids and in developing additional confidence in their ability to manage the care of patients with opioid use disorder would be a good addition to the guideline. Specifically, programs similar to the project BC ECHO on Substance Use, which provides guidance for primary care providers to assist patients with opioid use disorder, are valuable resources that should be included in clinical guidelines. Resources that support health care professionals to better understand how opioids can be used ultimately empower them to make the best evidence-based decisions confidently.

\section{Nilanga Aki Bandara BSc}

Teaching assistant, University of British Columbia, Vancouver, BC

\section{Vahid Mehrnoush MD}

Postdoctoral research fellow, University of British Columbia, Vancouver, BC

\section{Jay Herath PhD}

Instructor, University of British Columbia, Vancouver, BC

- Cite as: CMAJ 2020 November 2;192: E1369. doi: 10.1503/cmaj.76776

\section{References}

1. Tyndall M. A safer drug supply: a pragmatic and ethical response to the overdose crisis. CMAJ 2020;192:E986-7.

2. Vulnerable populations and COVID-19 - Canada.ca [Infosheet]. Ottawa: Public Health Agency of Canada; 2020. Available: www.canada.ca/en/public -health/services/publications/diseases-conditions /vulnerable-populations-covid-19.html (accessed 2020 Aug. 28).

3. von Gunten CF. The pendulum swings for opioid prescribing. J Palliat Med 2016;19:348.

4. Webster F, Rice K, Katz J, et al. An ethnography of chronic pain management in primary care: The social organization of physicians' work in the midst of the opioid crisis. PLoS One 2019;14:e0215148.

5. Comerci G Jr, Katzman J, Duhigg D. Controlling the swing of the opioid pendulum. N Engl J Med 2018;378:691-3.

6. Ahamad K, Bach P, Brar R, et al. Risk mitigation: in the context of dual public health emergencies [Guidance document]. Vancouver: British Columbia Centre on Substance Use; 2020. Available: www.bccsu.ca/wp-content/uploads/2020/04/Risk -Mitigation-in-the-Context-of-Dual-Public-Health -Emergencies-v1.5.pdf (accessed 2020 Aug. 28).

Competing interests: None declared. 